\title{
End-stage renal failure due to analgesic nephropathy, its changing pattern and cardiovascular mortality
}

\author{
F. P. Brunner and Neville H. Selwood, on behalf of the EDTA-ERA Registry Committee \\ Department für Innere Medizin, Universität Basel, Switzerland; and EDTA Registry, St Thomas' Hospital, London, UK
}

\begin{abstract}
The changing pattern of prevalence and age distribution of analgesic nephropathy as a cause of end-stage renal failure (ESRF) in patients on RRT was analysed using the EDTA-ERA Registry's files. Comparing 1990 to 1981 , the percentage of patients with analgesic nephropathy decreased in many European countries and the Registry's average came down from 3 to $2 \%$. The highest prevalence was noted for Switzerland, which showed a decrease from 28 in 1981 to $12 \%$ in 1990 . During the same interval the age distribution shifted to the right with an increase in median age from 57 to 63 at start of RRT for analgesic nephropathy. In Switzerland the age-specific acceptance rate to RRT for patients with analgesic nephropathy decreased to less than $1 / 3$ in the age cohorts below 55 but increased in those aged 65 or older. This increase in the elderly cohorts appeared to be related to the growing acceptance rate to RRT of elderly patients in general rather than to an increasing incidence of ESRF due to analgesic nephropathy. Mortality in general and death rates due to cardiovascular causes were found not to differ in RRT patients with analgesic nephropathy from that of other standard primary renal diseases (excluding diabetic nephropathy and systemic diseases). Some 20 years after withdrawal of phenacetin from the analgesic market, analgesic nephropathy all but disappeared as a cause of ESRF in Sweden and Denmark, and the same may be expected to occur in countries like Switzerland, Belgium, and others in the not too far distant future.
\end{abstract}

Key words: EDTA Registry; analgesic nephropathy; cardiovascular mortality on renal replacement therapy; epidemiology of end-stage renal failure due to analgesic nephropathy

\section{Introduction}

During the last three decades we have witnessed a decrease in the sale and abuse of mixed analgesic drugs

Correspondence and offprint requests to: Dr Neville Selwood, EDTA Registry, St Thomas' Hospital, London SEl 7EH, UK containing phenacetin in many European countries due to legal restriction, public education, and finally by the pharmaceutical companies removing phenacetin from mixed preparations [1-3]. All these measures should have affected the epidemiology of analgesic nephropathy, and the EDTA Registry's files afforded a unique opportunity to study any changes in the prevalence of ESRF caused by this condition. The Registry's files were also used to detect possible differences in overall and cardiovascular mortality on RRT, comparing analgesic nephropathy to standard primary renal diseases.

\section{Methods}

The methods of data collection used by the EDTA Registry have been described previously [4]. This report is based on data provided on individual patient questionnaires and relates to treatment up to 31 December 1990. The percentage of centres reporting their patients to the EDTA-ERA Registry varied between $50 \%$ and $100 \%$ for the countries mentioned specifically in this report. The analyses were carried out by the methodology developed by Dr Neville Selwood as previously described [5]. Age and sex specific acceptance rates to RRT were calculated for Switzerland, where patient questionnaire returns have been $80 \%$ to $90 \%$ complete and where the total number of new patients has been checked every year by telephone calls to non-reporting centres by FB.

\section{Results and comments}

\section{Prevalence and age distribution at start of $R R T$}

As judged by the proportion of patients starting RRT due to analgesic nephropathy and their age distribution, the prevalence of analgesic nephropathy has been changing in Europe. Comparing 1990 to 1981, the proportion with analgesic nephropathy as a percentage of all patients starting RRT decreased in most European countries (Figure 1). The highest proportion was noted for Switzerland, with $28 \%$ of patients in 1981 and a lower but still remarkable $12 \%$ in 1990. 


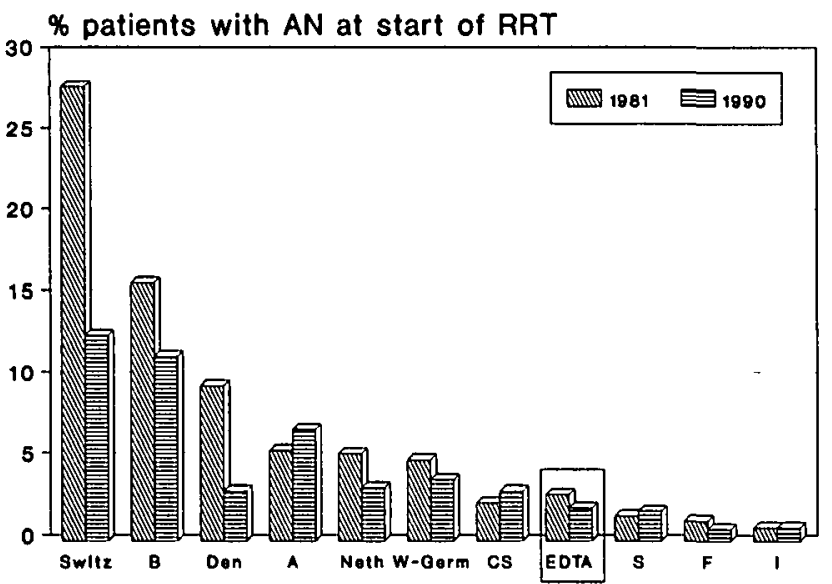

Fig. 1. Proportion of patients with analgesic nephropathy at start of RRT shown for the total registry (EDTA), for all countries above the EDTA average (Switzerland, Belgium, Denmark, Austria, Netherlands, Germany, Czechoslovakia) and for selected countries with lower proportions (Sweden is shown because of its high prevalence of analgesic nephropathy in the 1960s and France and Italy were selected as the two countries with the largest number of patients on RRT and of inhabitants).

For Belgium the percentage decreased from $16 \%$ to $11 \%$. In most other countries there was also a decrease to be noted comparing 1990 to 1981 . The EDTA average decreased from $3 \%$ in 1981 to $2 \%$ in 1990 . The proportion of patients with analgesic nephropathy accepted for RRT was recorded for every EDTA country in the 1988 combined report [6]. The percentage of patients accepted for RRT in 1987 with analgesic nephropathy for countries not listed in Figure 1 was $3.2 \%$ in Portugal, $2.9 \%$ in Cyprus, $2.7 \%$ in the German Democratic Republic, $1 \%$ in the United Kingdom, and less than $1 \%$ in all other countries [6].

Analgesic abuse has been more prevalent among females, and this is shown again as the female:male ratio for all patients starting RRT between 1970 and 1990 (Figure 2). The EDTA average was 2.7 females for 1 male, and Belgium had the highest preponderance of females with a ratio of 4.9 .

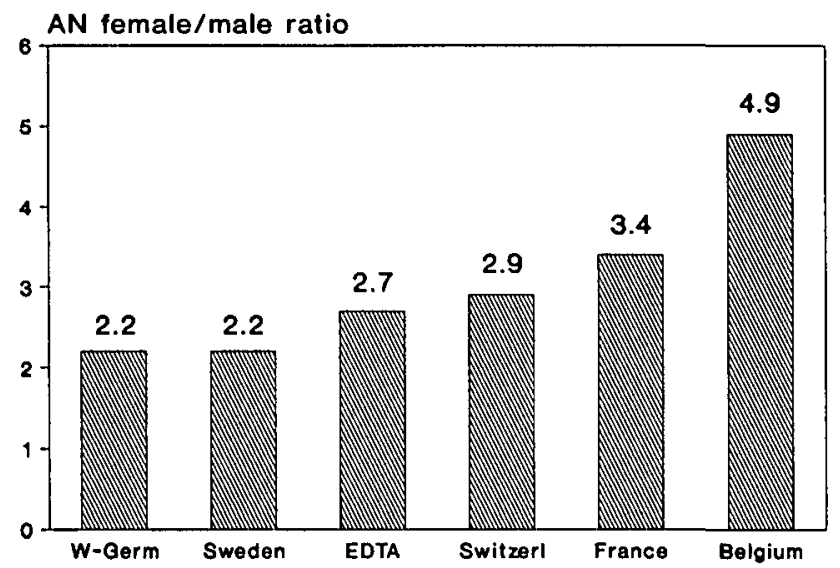

Fig. 2. Female:male ratio of patients starting RRT for end-stage renal failure due to analgesic nephropathy in 1970-1990, shown for selected countries.
The age distribution of all patients with analgesic nephropathy at start of RRT shifted to the right comparing 1990 to 1980 as shown in Figure 3. In 1980 some $30 \%$ or more were in each of the cohorts aged 45-54 and 55-64. In 1990 there were barely any patients aged below 35 , but over $30 \%$ were now in the age group 65-74 and 10\% above age 75 . Median age rose from about 57 in 1980 to 63 in 1990. Similar changes in age distribution have been reported from Australia [7].

The changing pattern of analgesic nephropathy can be appreciated best in Switzerland where this disease was first described in 1953 [8] and where it has had the highest prevalence worldwide over the last decades. The age-specific acceptance rate for Swiss patients with analgesic nephropathy is shown for 1980 and 1990 in Figure 4a (identical trends were observed regarding the age-specific acceptance rates in both sexes). In 1980 some 40 patients per million population aged 45-54 and about 60 patients p.m.p. aged 55-64 (or more than 100 per million females aged $55-64$ and over 30 per million males aged 55-64) were accepted for RRT with ESRF attributed to analgesic nephropathy. Ten years later, in 1990, not a single patient starting RRT for analgesic nephropathy was below age 35 , and very few were in the cohorts $35-44$ and 45-54. A definite decrease is also demonstrated for the cohort aged 55-64. However, the number of patients aged 65-74 more than doubled and there were some 10 per million population aged above 75 who started dialysis for ESRF due to analgesic nephropathy in 1990. Almost identical numbers and trends in the age-specific acceptance rates have been recorded in Australia between 1981 and 1991 [7].

One explanation for this increasing incidence in elderly patients could be the well-known fact that many patients discontinued analgesic abuse with phenacetin-containing mixed analgesics once renal disease had been detected or when phenacetin was more difficult to obtain. This could have lessened further damage to the kidneys and delayed the appearance of ESRF from age $40-50$ to age 70 or 80 . Another explanation

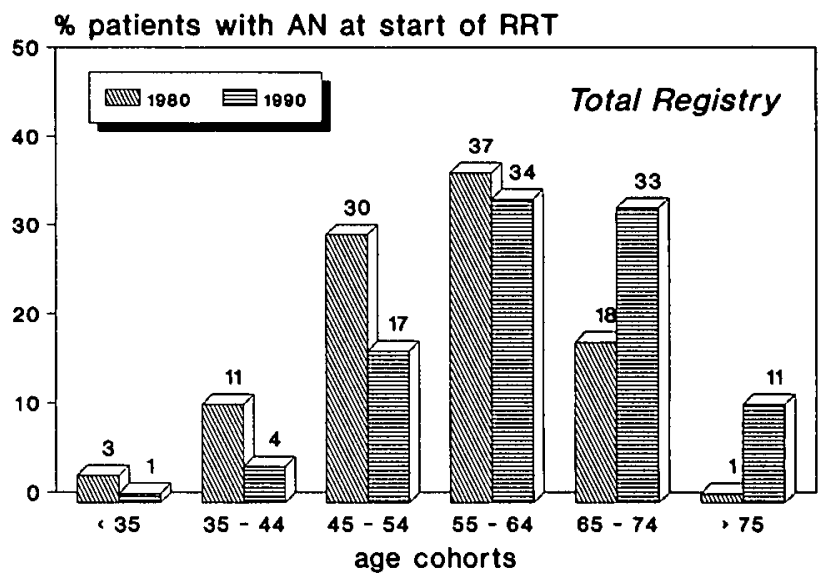

Fig. 3. Age distribution of patients starting RRT for end-stage renal failure due to analgesic nephropathy in 1980 and in 1990. 

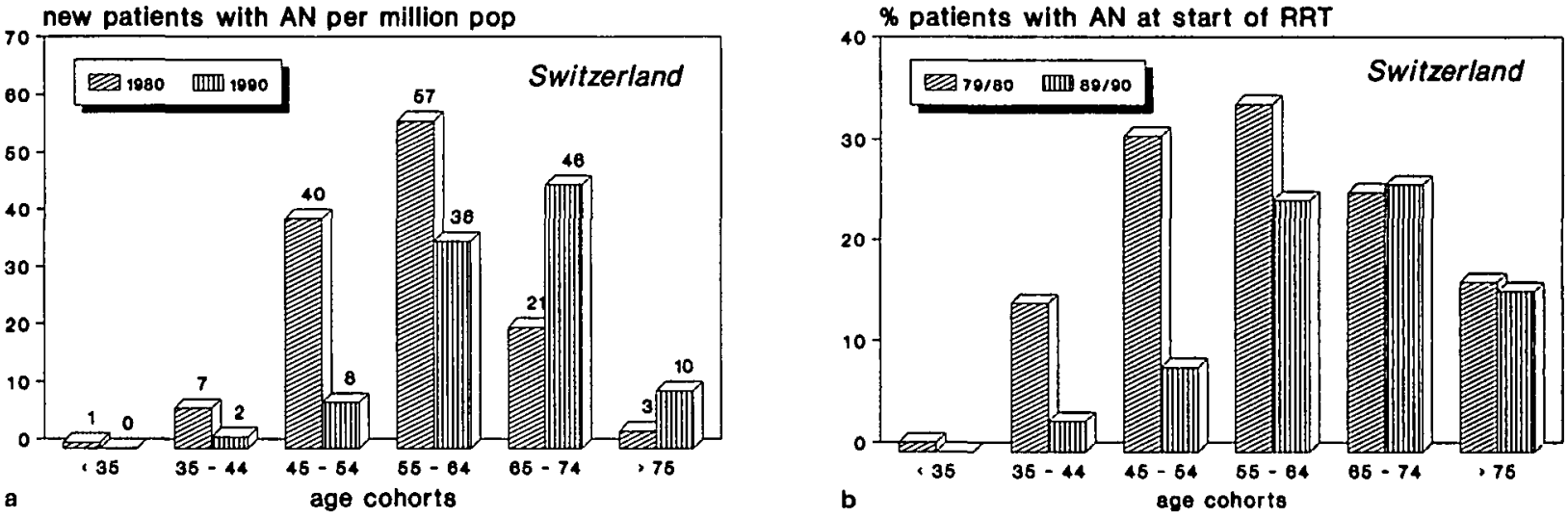

Fig. 4. End-stage renal failure due to analgesic nephropathy in Switzerland. a. Age-specific acceptance rate (per million general population of same age cohort); b. patients with analgesic nephropathy as a percentage of all patients accepted for for RRT.

is suggested by Figure $4 \mathrm{~b}$, which shows the percentage of patients with analgesic nephropathy as a proportion of all patients starting RRT in each age cohort separately for $1979 / 80$ and for $1989 / 90$. It is important to note that the percentage in the age group 65-74 and above 75 did not change over this 10 -year interval. There is no doubt, therefore, that the number of patients with end-stage renal failure due to analgesic nephropathy has been decreasing during the 1980s in Switzerland at least up to the age of 65 . In patients above the age of 65 , the true incidence of end-stage renal failure due to analgesic nephropathy has probably remained stable, and the increased acceptance rate was the result of an increased acceptance rate of elderly patients in general.

\section{Mortality and death rates due to cardiovascular causes}

Accelerated artherosclerosis with coronary, carotid, renal, and peripheral arterial occlusive disease has been reported to cause serious morbidity and premature mortality in patients with analgesic nephropathy, mainly from Australia [9] but also from Switzerland [10]. The study from Australia [9] compared patients with analgesic nephropathy to patients with glomerulonephritis accepted for RRT during the same 5-year period. Overall mortality was significantly higher in the former group with 50.8 versus $15.8 \%$. However, no details regarding age distribution and other prognostic features of the two patient groups were given. In the 20-year longitudinal study of initially healthy female factory workers without renal disease from Switzerland [10] a group with analgesic abuse was compared to a matched control group without abuse. The risk of renal or cardiovascular disease was increased in the group with analgesic abuse but endstage renal disease was a rare event. It seemed important therefore to analyse overall mortality and death rates due to cardiovascular causes in typically European patient cohorts on RRT with analgesic nephropathy in comparison to all patients with standard primary renal diseases (glomerulonephritis, pyelonephritis/interstitial nephritis, polycystic kidney diseases) as shown in Figures 5-8. These analyses were restricted to Belgium, The Netherlands, Germany, Switzerland, and Austria, i.e. countries with a sizeable proportion of patients with analgesic nephropathy. In contrast to the findings reported from Australia [9] mortality did not differ appreciably between the cohorts with analgesic nephropathy as compared to those with other standard primary renal diseases. Furthermore mortality in females with analgesic nephropathy was slightly lower compared to males, as has been reported for European patients with other common renal diseases [11].

Cardiovascular mortality including death rates due to myocardial ischaemia and infarction have long been known to be increased in patients on RRT as recorded again in recent EDTA reports $[11,12]$. Of particular interest were the geographical differences in the coronary death rates on RRT, which reflected the pattern in the general population of higher death rates due to myocardial ischaemia and infarction in Northern versus Southern Europe. These geographical differences made it important to restrict the analyses of cardiovascular death rates in patients with analgesic nephropathy to countries in central Europe with similar risks of cardiovascular death in the general population. Death rates due to myocardial ischaemia and infarction (Figure 6) were slightly higher in male patients than in female patients but did not differ appreciably between patients with analgesic nephropathy as compared to patients with other primary renal diseases. The same was true for other cardiac causes (Figure 7). Lethal cerebrovascular accidents (Figure 8) had not only a similar incidence in males compared to females but also occurred at similar rates in patients with analgesic nephropathy.

Hence we may conclude that patients on RRT due to analgesic nephropathy have an overall mortality and cardiovascular death rates related to end-stage renal failure in general, as observed in other patient groups with standard primary renal diseases.

\section{Conclusions regarding the incidence of analgesic nephropathy as a cause of end-stage renal failure}

The sale of phenacetin-containing mixed analgesics was discontinued in the early 1960s in Sweden [1]. The 

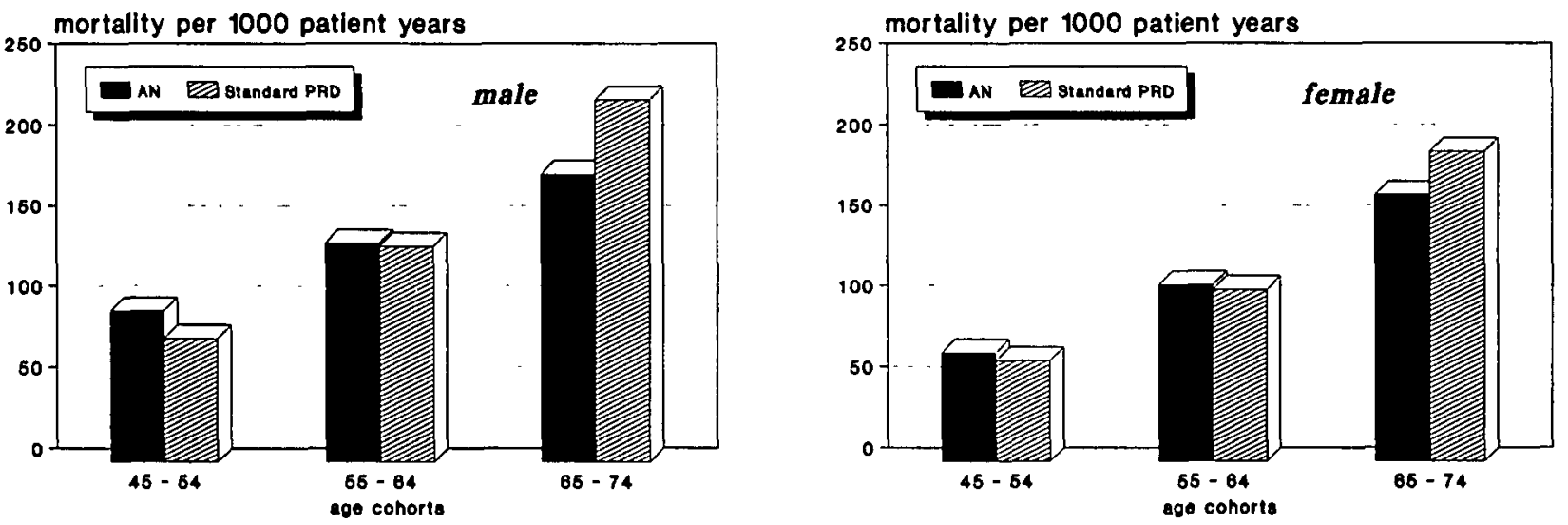

Fig. 5. Average yearly mortality during the first 5 years of RRT in patients starting treatment in 1980-1985 in Austria, Belgium, FRG, Netherlands, and Switzerland. Mortality per thousand patient years at risk is shown separately for patients with analgesic nephropathy (AN) and for patients with all other standard primary renal diseases.

\section{Myocardial Ischaemia and Infarction}
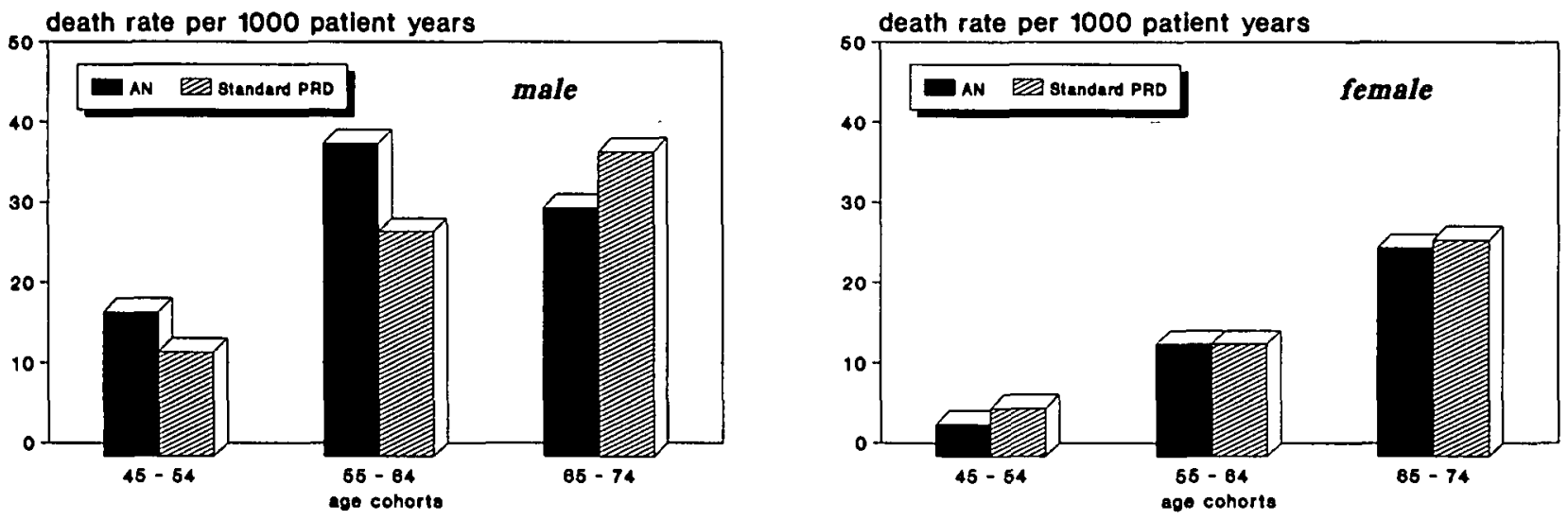

Fig. 6. Average annual death rates per 1000 patient years due to myocardial ischaemia and infarction (same patient groups, countries, and periods of time as Figure 5).

\section{Cardiac (excl MI)}
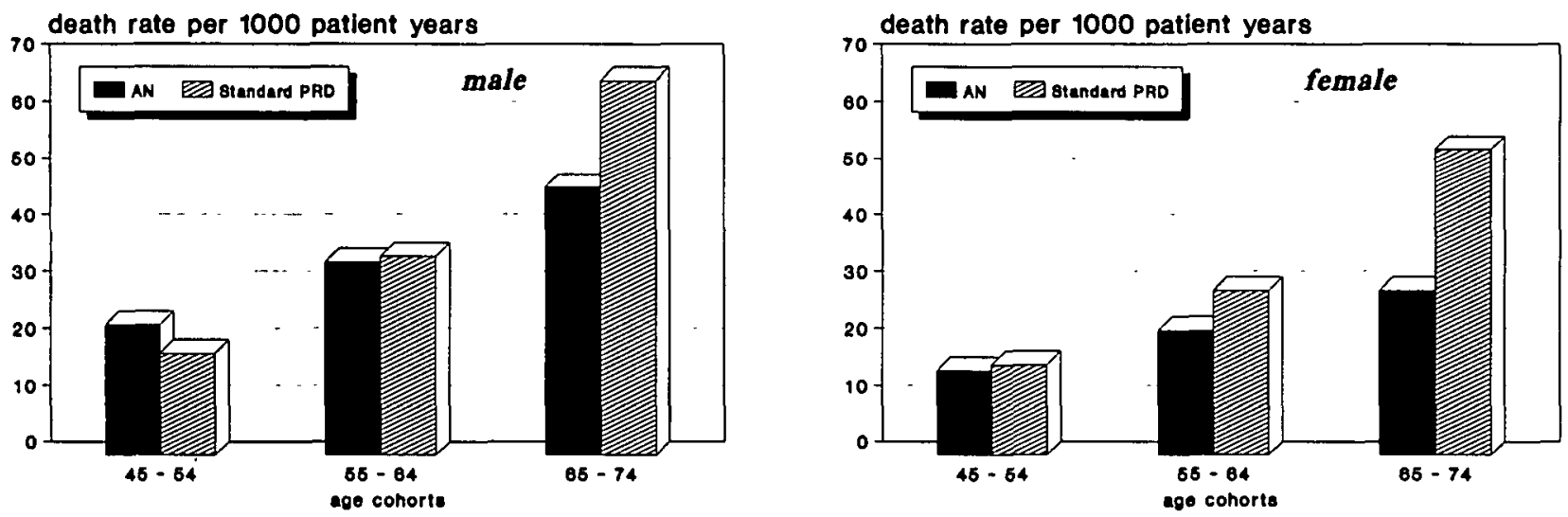

Fig. 7. Average annual death rates per 1000 patient years due to cardiac causes excluding myocardial ischaemia and infarction (same patient groups, countries, and periods of time as Figure 5).

proportion of patients with analgesic nephropathy as a cause of end-stage renal failure in Sweden had probably decreased slightly by 1970 , with $10 \%$ of all patients accepted for RRT at that time, and this decreased further to $2 \%$ in 1980 (Figure 9). Analgesic nephropathy has now almost disappeared in Sweden and there was only a small number of old patients starting RRT for analgesic nephropathy in 1990 in 


\section{Cerebro-Vascular Accident}
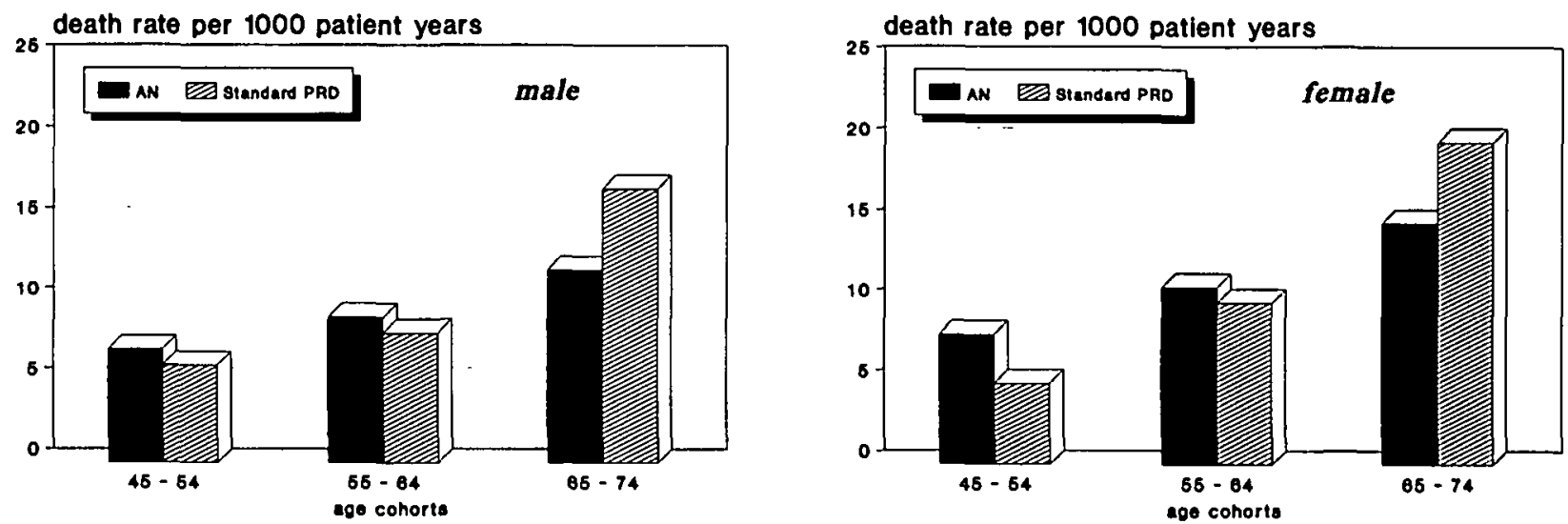

Fig. 8. Average annual death rates per 1000 patient years due to cerebrovascular accident (same patient groups, countries, and periods of time as Figure 5)

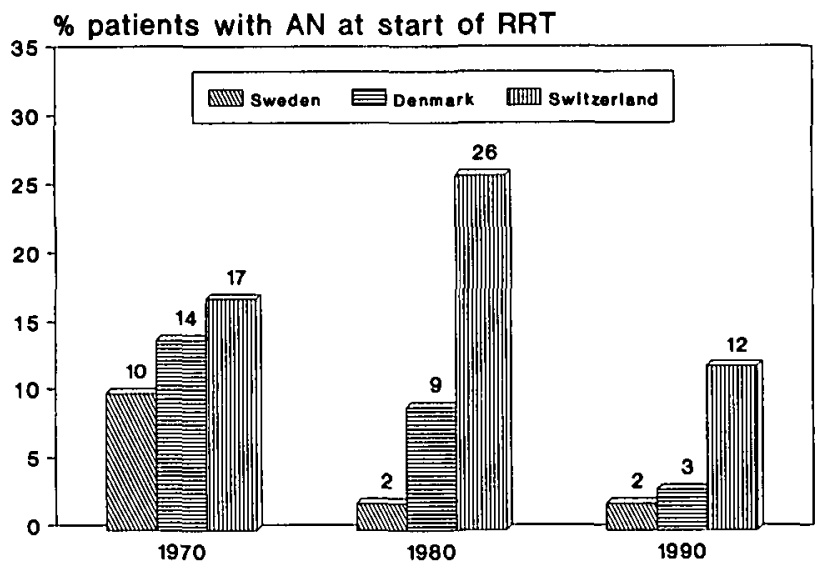

Fig. 9. Proportion of patients with analgesic nephropathy at start of RRT in Sweden, Denmark, and Switzerland in 1970, 1980, and 1990.

Sweden. In Denmark, the restriction to the sales of phenacetin-containing mixed analgesics came into effect during the early 1960s but phenacetin continued to be available on prescription only until 1984 . The percentage of Danish patients with analgesic nephropathy at start of RRT decreased from $14 \%$ in 1970 to $9 \%$ in 1980 and was as low as $3 \%$ in 1990 . In Switzerland the highest proportion was recorded in 1980 and 1981 with some $26-28 \%$ of patients starting RRT during those years. Phenacetin has been disappearing rather slowly from the market in Switzerland and was still available in a few mixed analgesics in the late 1980s. We may expect that some 10-20 years hence analgesic nephropathy will no longer be a significant cause of end-stage renal failure in Switzerland and other countries as was the case in Sweden some 20 years after the disappearance of phenacetin.

Acknowledgements. This work was supported by grants from the governments and National Societies of Nephrology of Austria, Cyprus, Denmark, Finland, France, Germany, Iceland, Ireland, Israel, The Netherlands, Norway, Switzerland, and United Kingdom. Grants were also made by the AKZO Group (Fibres and Polymers
Division), Asahi Medical Co. Ltd., Baxter Healthcare Corporation, Bellco SpA, Braun Melsungen AG., Cilag AG Research, Cobe International Ltd., Fresenius Ltd., Gambro, Hospal Ltd, and Sandoz AG.

We gratefully acknowledge the collaboration of the National registries of Bulgaria, Hungary and the Netherlands (Renine) and the regional registries of Lombardy, Italy, Catalunia, and Andalucia, Spain, in providing patient data to the EDTA Registry. The co-operation of UK Transplant Service, Bristol, United Kingdom is also acknowledged.

We thank those doctors and their staff who have completed questionnaires. Without their collaboration, this report could not have been prepared.

The hard work and dedication of the staff of the EDTA Registry: Dr Elizabeth Jones, Mrs Lynne Saker, Miss Tamara Sobel, and Mrs Linda Carter, in the preparation of this report is gratefully acknowledged.

\section{References}

I. Bengtsson U. Analgetika-Nephropathie-Langfristige Erfahrungen aus Schweden. Zeitschr Urol Nephrol 1989; 82: $121-125$

2. Pommer W, Glaeske G, Molzahn M. Analgetika-Verbrauch in der Bundesrepublik Deutschland. Deutsch Med Wochemschr 1987; 112: 787-790

3. Elseviers MM., De Broe ME. Analgesic nephropathy in Belgium is related to the sales of particular analgesic mixtures. Nephrol Dial Transplant 1994; 9: 41-46

4. Demography of dialysis and transplantation in Europe, 1984. Report from the European Dialysis and Transplant Association Registry. Nephrol Dial Transplant 1986; 1: 1-8

5. Kramer P, Broyer M, Brunner FP et al. Combined Report on Regular Dialysis and Transplantation in Europe, XIV, 1983. Proc Eur Dial Transplant Assoc-ERA 1985; 21: 2-68

6. Tufveson G, Geerlings W, Brunner FP et al. Combined report on regular dialysis and transplantation in Europe, XIX, 1988. Nephrol Dial Transplant 1989; 4 [Suppl 4]: 5-29

7. Australia and New Zealand Dialysis and Transplant Registry. Disney APS, ed. ANZDATA Report 1992. Adelaide, South Australia

8. Spühler O, Zollinger HU. Die chronisch-interstitielle Nephritis. Z Klin Med 1953; 151: 1-49

9. Nanra RS. Analgesic nephropathy: etiology, clinical syndrome, and clinicopathologic correlations in Australia. Kidney Im 1978 13: $79-92$ 
10. Dubach UC, Rosner B, Sturmer T. An epidemiologic study of abuse of analgesic drugs. Effects of phenacetin and salicylate on mortality and cardiovascular morbidity, (1968 to 1987). $N$ Engl J Med 1991; 324: 155-160

11. Fassbinder W, Brunner FP, Brynger $\mathrm{H}$ et al. Combined Report on Regular Dialysis and Transplantation in Europe, XX, 1989. Nephrol Dial Transplant 1991; 6 [Suppl 1]: 5-35

12. Raine AEG, Margreiter R, Brunner FP et al. Report on Management of Renal Failure in Europe, XXII, 1991. Nephrol Dial Transplant 1992; 7 [Suppl 2]: 7-35

Received: 2.12 .93 